\title{
ANÁLISIS DE INTERESES AMBIENTALES MEDIANTE LÓGICA DIFUSA EN LA RESERVA FORESTAL REGIONAL DEL NORTE DE BOGOTÁ-COLOMBIA
}

\section{ANALYSIS OF ENVIRONMENTAL CONCERNS THROUGH FUZZY LOGIC: IF REGIONAL FOREST RESERVE IN THE NORTH OF BOGOTÁ-COLOMBIA}

\begin{abstract}
Adriana Posada ${ }^{1}$
${ }^{1}$ Economista Agrícola, Esp. Gestión Social y Ambiental. M.Sc. Planeación Urbana y Regional. Docente Facultad Ingeniería Geográfica y Ambiental, Universidad de Ciencias Aplicadas y Ambientales U.D.C.A, Calle 222 No. 55-37, Bogotá, Colombia. aposada@udca.edu.co
\end{abstract}

Rev. U.D.C.A Act. \& Div. Cient. 15(2): 437 - 446, 2012

\section{RESUMEN}

En Bogotá, Distrito Capital de Colombia, a pesar de ocurrir una expansión urbana, que sin considerar las condiciones naturales, se declaró como reserva forestal regional a una zona en el norte de la ciudad, en límites con otros municipios, que están respondiendo, paulatinamente, a la alta demanda de vivienda. La existencia de esta reserva forestal, en medio de la presión de los urbanizadores, ha generado un conflicto de intereses, durante más de una década. El conflicto se profundiza, ya que, por una parte, la información sobre los intereses reales de los diferentes grupos es imprecisa y volátil y, por otra, las acciones sobre el uso, el manejo y la ocupación del suelo, se deben definir y llevar a cabo, por parte de los entes encargados. Con el fin de aportar datos para observar esta situación, se adelantó una investigación, que tuvo como resultado la aplicación de una metodología alternativa, para establecer los intereses ambientales respecto a la reserva. La lógica difusa es el método que mejor se ajusta al análisis de intereses ambientales, en tanto, permite flexibilidad en las mediciones de las respuestas y amplía el horizonte de la gestión socioambiental, considerando diversos grupos de trabajo, para poder desarrollar un plan de manejo ambiental, más acertado en zonas de alta complejidad.

Palabras clave: Intereses ambientales, expansión urbana, conflicto ambiental.

\section{SUMMARY}

In Bogotá, capital of Colombia, due to its urban expansion, which does not consider the natural conditions, a regional forest reserve in the northern area of the city, limiting with other municipalities that are gradually responding to the high demand for housing, was declared. The existence of this forest reserve in the midst of pressure of housing development has created a conaflict of interest for over a decade. The conflict deepens since, on one hand, the information about the real interests of the different groups is imprecise and volatile and on the other one, actions on the use, management and land use must be defined and carried out by the responsible authorities. In order to provide data to monitor this situation, a research project was carried out, resulting in the implementation of an alternative methodology to establish the environmental concerns with respect to the reserve. Fuzzy logic is the method best suited to the analysis of environmental concerns, insofar as it allows flexibility in the measurements of responses and expands the horizon of the socio-environmental action, taking into account various working groups, in order to develop a more successful environmental management plan in areas of high complexity.

Key words: Environmental concerns, urban sprawl, environmental conflict.

\section{INTRODUCCIÓN}

Colombia, se caracteriza por ser un país heterogéneo en su geografía, con diferentes niveles de desarrollo regional y con condiciones culturales y sociales diversas (DNP, 2010). Su capital, Bogotá, de acuerdo con la Alcaldía Mayor (2012), registra una población de 7.467.804 habitantes, que representan el $16 \%$ de la población de la Nación; la alta tasa de crecimiento ha generado una ocupación de 36.364ha, de 
las 38.431 del suelo urbano, a un ritmo de 326ha/año, logrando una densidad poblacional de $19.388 \mathrm{hab} . / \mathrm{km}^{2}$ y una densidad habitacional de 5.354 viviendas $/ \mathrm{km}^{2}$. Este proceso refleja un cambio en la concepción del valor de la tierra, en el que se ha privilegiado la urbanización sobre otros tipos de usos del suelo, como la agricultura o la conservación. Esta condición, se evidencia en la zona norte de Bogotá, donde, según Acevedo et al. (2000), debido a las características particulares de sus geoecosistemas y a su papel en la dinámica del sistema ecológico regional, el impacto de las decisiones de expansión es mayor que en cualquiera otra de las zonas de expansión autorizadas.

El IEU (2011) informó que, en el 2000, por falta de concertación entre la Corporación Autónoma Regional de Cundinamarca CAR y el gobierno distrital, respecto al Plan de Ordenamiento Territorial de Bogotá, el Ministerio de Medio Ambiente expidió la Resolución 475, por medio, de la cual, ordenó a la CAR delimitar y desarrollar el Plan de Manejo, de la que se denominó "Reserva Forestal Regional del Norte de Bogotá" (RFRNB); no obstante, la sentencia, la declaración de esta reserva, se interrumpió por más de una década, debido a la objeción de grupos sociales de interés, de los cuales, según el IEU (2011), se destacan, por una parte, los intereses de quienes insisten en que esta zona se debe dedicar a la expansión urbana y, por la otra, los intereses de los que creen que debe ser una zona de conectividad y de protección de la estructura ecológica principal. Algunos investigadores, como Ardila (2009), explican que la demora en la declaratoria de la RFRNB es un ejemplo de los conflictos interinstitucionales y de las complejidades surgidas entre las acciones de gobierno y la dinámica social y política, en la cual, los juegos de intereses determinan la dirección de los procesos, aún a costa de acuerdos concretados en la normatividad.

En el enfoque de Carrizosa (2006), la sostenibilidad integral potencial es una característica de la totalidad de un territorio, que plantea la necesidad de construir metodologías capaces de estudiar complejamente esa totalidad. En esa tendencia, ve la necesidad de considerar que sus objetivos surgen de percepciones individuales sobre las prioridades del ambiente y la sostenibilidad. Es aquí, donde se requiere aplicar la metodología que ofrece la lógica difusa, pues conforme la complejidad de un sistema aumenta nuestra capacidad para ser precisos y construir instrucciones sobre su comportamiento, disminuye hasta el umbral más allá, del cual, la precisión y el significado son características excluyentes (Morales, 2002).

La lógica es la razón, el principio que gobierna al universo (Aznar \& Alarcón, 2006). Para D’Negri \& De Vito (2006), es un conjunto de reglas usadas para generar inferencias creíbles en el marco del razonamiento exacto, es decir, es una lógica dicotómica o binaria, que admite dos posibilidades: verdadero o falso $(\mathbf{1}$ o $\mathbf{0})$, mientras que, la lógica difu- sa (fuzzy logic), es una metodología que proporciona una manera de obtener conclusiones, a partir de información de entrada vaga, ambigua, imprecisa, con ruido o incompleta; esta atenta a la aceptación de la imprecisión en el mundo real, especialmente, en la percepción y en el pensamiento humano; la lógica difusa, se basa en el principio que todo es cuestión de grado. La lógica difusa también es denominada "lógica borrosa". Mediante la lógica borrosa es posible valorar impactos ambientales a partir del conocimiento, generalmente impreciso y subjetivo, de una serie de características que componen la esencia de dichos impactos; empleando técnicas de razonamiento aproximado, se puede traducir la estimación borrosa de cada característica, llevar a cabo la agregación de dichas valoraciones y obtener un valor global del impacto (Peche, 2006). Adicionalmente, como lo expresa Durán (s/f), los sistemas borrosos permiten modelar cualquier proceso no lineal y aprender de los datos, haciendo uso de determinados algoritmos de aprendizaje.

Particularmente, el interés ambiental sobre la RFRNB contiene una incertidumbre de mayor o de menor grado y para obtener precisión al respecto, es necesario analizar la situación como un proceso complejo (no lineal), frente, al cua, no existen modelos absolutos, pero sí es posible admitir valores cuantificando la incertidumbre, lo difuso del interés ambiental. Con base en lo explicado por D 'Negri \& De Vito (2006), lo que se busca, mediante el empleo de la lógica difusa, es describir y formalizar una realidad, aplicando modelos flexibles que interpretan las leyes del comportamiento humano y las relaciones entre las personas. Para describir tal realidad incierta es necesario valerse de elementos que pueden ser nítidos, pero también difusos. En el caso de la RFRNB, utilizando la lógica difusa, se busca demostrar cómo y porqué el interés ambiental con más peso o con mayor grado de certeza, será el mejor para decidir el camino a seguir.

\section{MATERIALES Y MÉTODOS}

La aplicación de la lógica difusa, para establecer intereses ambientales, demuestra el paso de métodos tradicionales a alternativos, para obtener conocimiento frente a un caso de complejidad, que amerita contar con información adicional, útil para dirimir con mayor certeza los conflictos socioambientales. Por ello, en primera instancia, se establecieron los nudos críticos de la situación compleja, que están ligados a asuntos de intereses así: Sobre el qué: No hay claridad entre los intereses individuales y los colectivos. Sobre los recursos: Los recursos son los bienes y los servicios ambientales; en este caso, el recurso de mayor interés es el suelo, como soporte de actividades y de ocupación. Sobre el lugar: La zona del conflicto es para unos exclusivamente del distrito y, para otros, por sus características geoecosistémicas, es de la región. Sobre el quién: Los entes más influyentes en las decisiones pueden ser los grupos sociales que habitan 
la zona, el D. C., la CAR, la Procuraduría, el Ministerio de Ambiente y Desarrollo Sostenible. Sobre el tiempo: Situación que se mantiene, con sus variantes, data de ayer, es vigente hoy y continúa con tendencias hacia el mañana, pero por la urgencia de lograr armonía ambiental, se convierte en una situación que está en límite sobre el tiempo. De estos intereses, se destacaron como las restricciones más relevantes, las siguientes:

- De autoridad: quién manda a quién en materia ambiental: ¿El Distrito, la Procuraduría General de la Nación, el Ministerio de Ambiente y Desarrollo Sostenible, la CAR? Y en materia de desarrollo: ¿Planeación Nacional, el Distrito Capital, la Localidad de Suba? Y en cuanto a desarrollo urbano: ¿La Lonja de Propiedad Raíz, la Cámara de Comercio, la Curaduría Urbana? En suma, cquién direcciona los aspectos ambientales del desarrollo urbano-regional?

- De aprovechamiento de la renta del suelo: este es el interés de mayor peso, que se expresa en contravía frente a los intereses generales sobre la RFRNB, pues la velocidad de ocupación del suelo para viviendas de estratos altos es lo que predomina en las zonas vecinas a la RFRNB, evidenciando un interés de particulares.

- De tipo espacial: porque el uso, el manejo y la ocupación de la RFRNB impacta positiva o negativamente a toda la Nación y porqué la zona está ubicada en coordenadas del Distrito, pero al mismo tiempo, está asociada a toda la región de la sabana (especialmente, con los municipios vecinos de Bogotá), debido a que hace parte de su estructura ecológica principal.
Para trasladar estos interrogantes a la población, el conjunto de la muestra fue el $42 \%$ del total del universo, que está representado en personas del interior de la reserva (interesados intrínsecos) y también en su exterior inmediato (interesados extrínsecos), lo que equivale a 65 entrevistados. Para los interesados extrínsecos, se abordaron personas de la zona rural de la Localidad de Suba de Bogotá y a personas en los municipios vecinos de Cota, de Chía, de Cajicá y de Sopó, debido a que el ámbito regional, desde la óptica de los municipios vecinos, permite ver "la otra cara de la moneda".

Se diseñó una entrevista semiestructurada, con 15 preguntas, que condujeran, gradualmente, hacia la obtención de datos relacionados con las variables del método fuzzy logic. La aplicación de cada entrevista, se georeferenció en la cartografía, obtenida a partir de la aprobación oficial de la RFRNB, por parte de la CAR (2011), quedando el polígono tal como se ubica en el mapa 1 , donde se aprecia la zona de estudio.

La entrevista, se diseñó utilizando un flujograma, en el que se concentra la atención en tres preguntas claves, que permiten determinar el interés que existe por la RFRNB, con las siguientes tres variables:

Disponibilidad a agruparse o unión de esfuerzos (UF): La intención y la posibilidad de trabajar en grupo demuestra que, en temas ambientales, "la unión hace la fuerza"; cuando el trabajo es en grupo existe la necesidad de consensuar, de distribuir los esfuerzos y, en este orden de ideas, de "repartir

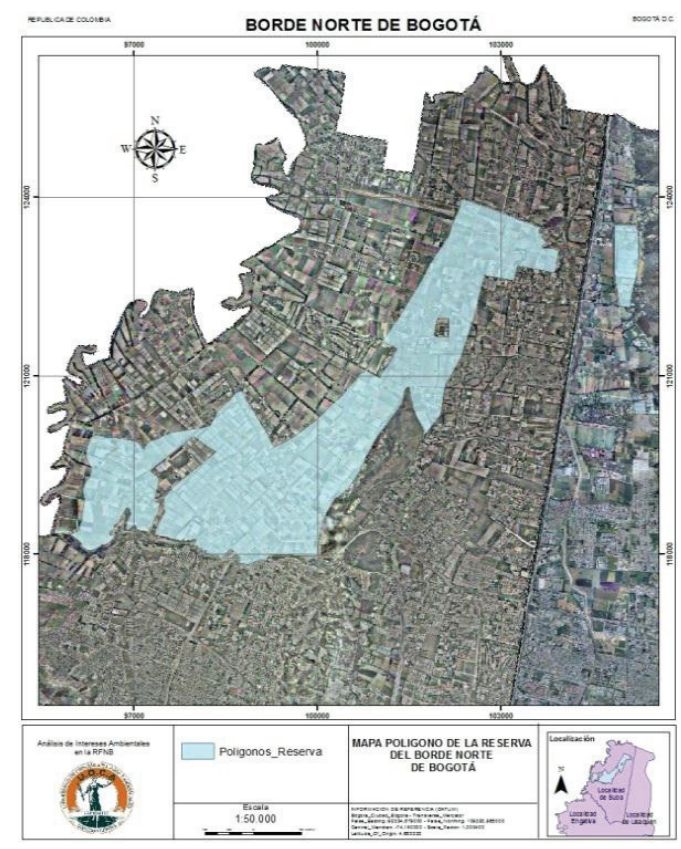

Mapa 1. Inserción de los datos sobre la declaratoria de reserva forestal de la CAR, en la base SIG del proyecto U.D.C.A (Romero, 2012). 
las ganancias", incluyendo beneficios para personas que no se conocen entre sí y que aún no han nacido. Disponibilidad de tiempo o temporalidad de las acciones (TDLA): Gran parte de la convicción sobre lo que se hace está determinada por el tiempo de dedicación que se le asigna a tal acción, por ello, si alguien está verdaderamente interesado en algo, el "invertir" tiempo en su realización pasa a ser un placer. Disponibilidad a pagar (DAP) o apoyo monetario: Posada (1997) explica que un mercado es una institución de intercambio, que sirve para desarrollar la actividad económica de la sociedad. Para ello, la valoración contingente es un intento de asignar un valor cuantitativo y monetario a los bienes y a los servicios suministrados por los recursos o sistemas ambientales, sea que se cuente o no, con precios de mercado (Azqueta, 1994). Al respecto, Mendieta (2005) afirma que la valoración contingente, estima cambios en el bienestar económico, derivados de cambios, en los niveles disponibles de calidad y de cantidad ambiental.

En síntesis, si una persona tiene la capacidad de trabajar en grupo, por siempre y, adicionalmente, está dispuesta a pagar para que se cumpla el fin de la RFRNB, está demostrando un interés real y no un interés difuso.

\section{RESULTADOS Y DISCUSIÓN}

Al aplicar la lógica difusa, se abre la posibilidad de entender que existen otras personas interesadas en la RFRNB, pero con vaguedad en su posición; esta vaguedad también cuenta cuando se trata de asuntos ambientales, porque no es obstáculo en la construcción de un mejor futuro, pero sí requieren mecanismos y estrategias para ser potenciados hacia la superación del conflicto ambiental.

Particularmente, para el caso de las posiciones (intereses) de las distintas autoridades, en la tabla 1 , se resumen los respectivos argumentos a favor de alguna posición sobre la RFRNB. Se observó, además, que desde la óptica del constructor existe mucho suelo disponible, oferta que al ser cruzada contra la demanda de vivienda, da como resultado una fuerte dinámica en el mercado inmobiliario, añadiendo, como valor agregado, las características ambientales de la zona. En esta situación, el urbanizador aprovecha la demanda de los ciudadanos por contar con lugares "tranquilos y sanos" para vivir y por los que están dispuestos a pagar un excedente, asunto que evidencia el diario El Tiempo (2012), al ofrecer proyectos urbanísticos, ubicados en zona forestal, como ventajas de vivir en las afueras de Bogotá.

Tabla 1. Síntesis de argumentos institucionales respecto a la RFRNB.

\begin{tabular}{|c|c|c|}
\hline ENTE & ARGUMENTOS & A FAVOR DE \\
\hline Misión (panel) de expertos & $\begin{array}{l}\text { Constituye la necesidad más apremiante de la zona, pues per- } \\
\text { mite la conexión de los pequeños parches de bosque entre sí } \\
\text { y los flujos de vida entre los cerros orientales y el río Bogotá, } \\
\text { asegurando su restauración y conservación en el tiempo }\end{array}$ & $\begin{array}{l}\text { Conectividad ecológica entre los cerros } \\
\text { orientales y el río Bogotá (Ârea Forestal) }\end{array}$ \\
\hline $\begin{array}{l}\text { Ministerio de Ambiente, } \\
\text { Vivienda y Desarrollo } \\
\text { Territorial }\end{array}$ & $\begin{array}{l}\text { Beneficio ambiental de orden nacional, apoyado por la Resolu- } \\
\text { ción } 475 / 2000 \text { y } 621 / 2000\end{array}$ & $\begin{array}{l}\text { Reserva Forestal Regional del Norte } \\
\text { (RFRN) }\end{array}$ \\
\hline CAR & $\begin{array}{l}\text { A pesar que existe una alta transformación del área }(96,4 \%) \\
\text { frente a la baja presencia de áreas naturales }(2,5 \%) \text {, se destaca } \\
\text { su gran importancia para Bogotá y la región, en virtud de sus } \\
\text { valores de conservación. Acuerdo \#011/2011 }\end{array}$ & $\begin{array}{l}\text { Reserva Forestal Regional Productora del } \\
\text { Norte de Bogotá, D.C. Thomas Van der } \\
\text { Hammen. }\end{array}$ \\
\hline Distrito Bogotá & $\begin{array}{l}\text { Decretos 619/200, 1110/2000, 469/2003, 190/2004 y 043/2010 } \\
\text { (Plan zonal del norte). Es al distrito a quien le corresponde in- } \\
\text { corporar los esquemas de protección en su territorio, indepen- } \\
\text { diente que tengan un carácter regional. El manejo del territorio } \\
\text { Bogotano es de potestad del Distrito. } \\
\text { Se destaca el Plan de Desarrollo de Bogotá 2012-2016, "Bo- } \\
\text { gotá Humana" }\end{array}$ & $\begin{array}{l}\text { Clasificación del suelo distrital en: zona } \\
\text { de expansión urbana, suelo Rural y suelo } \\
\text { de Protección. } \\
\text { Incorporó la Reserva Forestal Regional } \\
\text { del Norte de Bogotá Thomas van der } \\
\text { Hammen en la política de gestión am- } \\
\text { biental. }\end{array}$ \\
\hline
\end{tabular}


Antes de aplicar la lógica difusa y con el fin de comparar metodologías, se aplicaron análisis tradicionales para interpretar los resultados de las entrevistas. Al respecto, se pudo constatar que en un porcentaje importante, el interés manifestado por la RFRNB es superficial. En el gráfico 1, se pre- sentan los resultados distribuidos en proporciones, donde se aprecia que, en términos generales, la mayoría de las personas relacionadas con la zona, no están interesadas en la RFRNB como tal, pues sus intereses son variables, dispersos y poco puntuales al respecto.

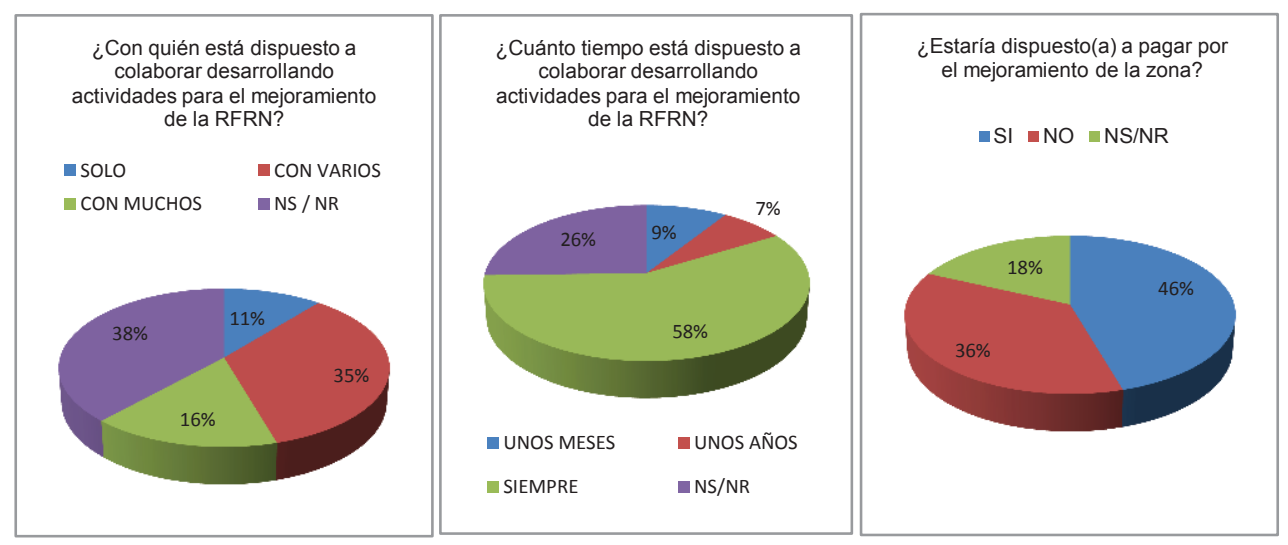

Gráfico 1. Distribución porcentual de las respuestas sobre "Unión de esfuerzos”, "Temporalidad de las acciones” y "Disponibilidad a pagar".

Adicionalmente, en el flujograma del gráfico 2, se observa la agrupación de los subconjuntos, según la tipología del interés. Aplicando un sistema de asociación de respuestas, se obtienen tres subgrupos del total de interesados intrínsecos, un subgrupo de interés sobre la RFRNB, otro de interés difuso y otro de interés real. Con este sistema, se inicia la demostración del interés por la RFRNB.

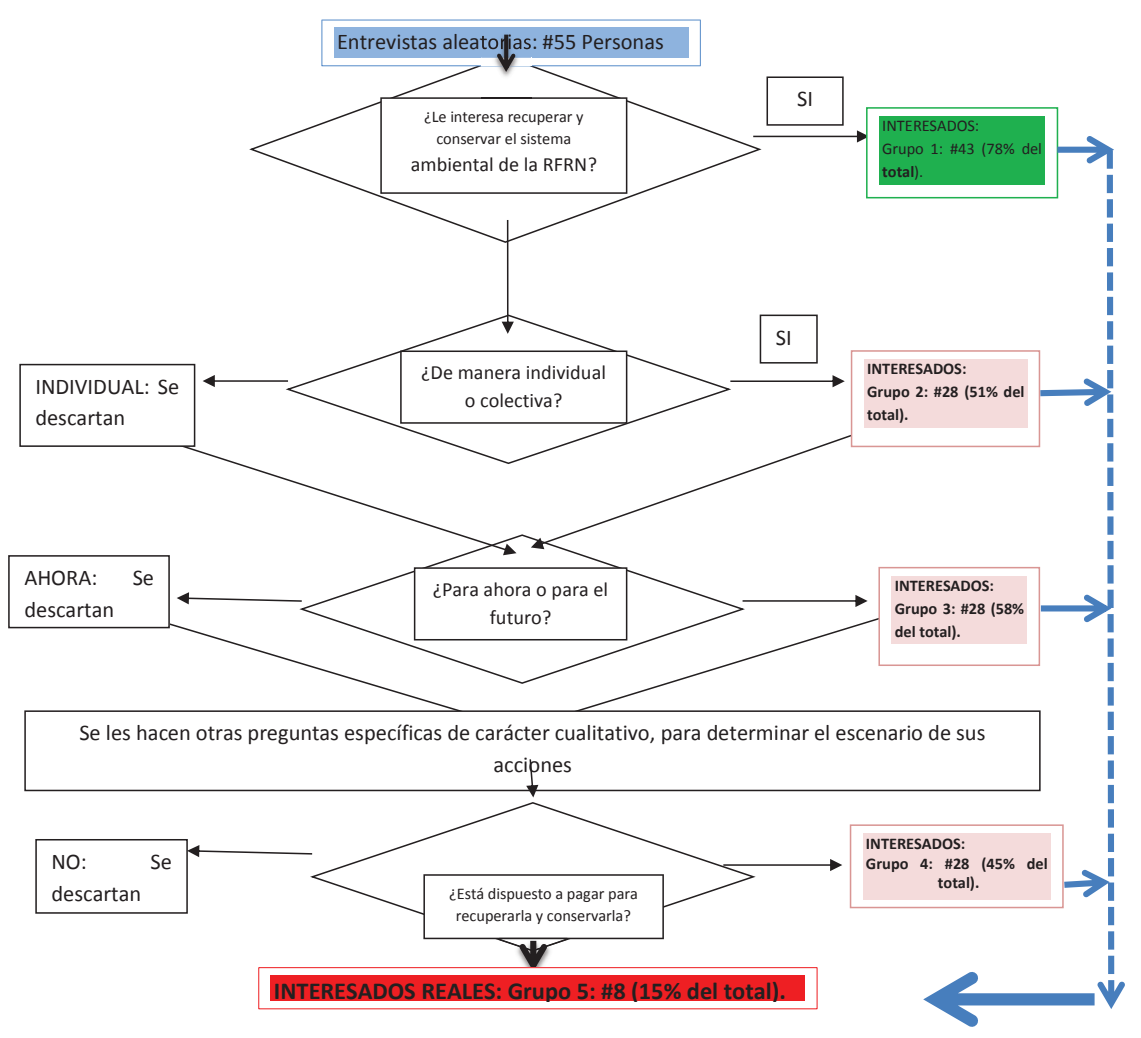

Gráfico 2. Flujograma de conjuntos (difusos y real) de interés en la RFRNB. 
El resultado que se observa en el gráfico 3, no es sorprendente; para muchos podría significar que un $15 \%$ de interés real en la RFRNB es bajo, pero en realidad es una cifra considerable, pues si se aplicara este mismo ejercicio en otro lugar de Bogotá, de Colombia o del mundo, la cifra podría ser menor, debido a que las personas, en su mayoría, no tienen un interés ambiental claro y real sobre su entorno.
Desde otro ángulo, el hecho de demostrar que un 15\% de la población cuenta con un interés real sobre la RFRNB, se interpreta como una ganancia, porque se está evidenciando que en esta zona Sí existen personas con quienes contar, para tejer un mejor futuro, no solamente para ellos, sino para el colectivo.
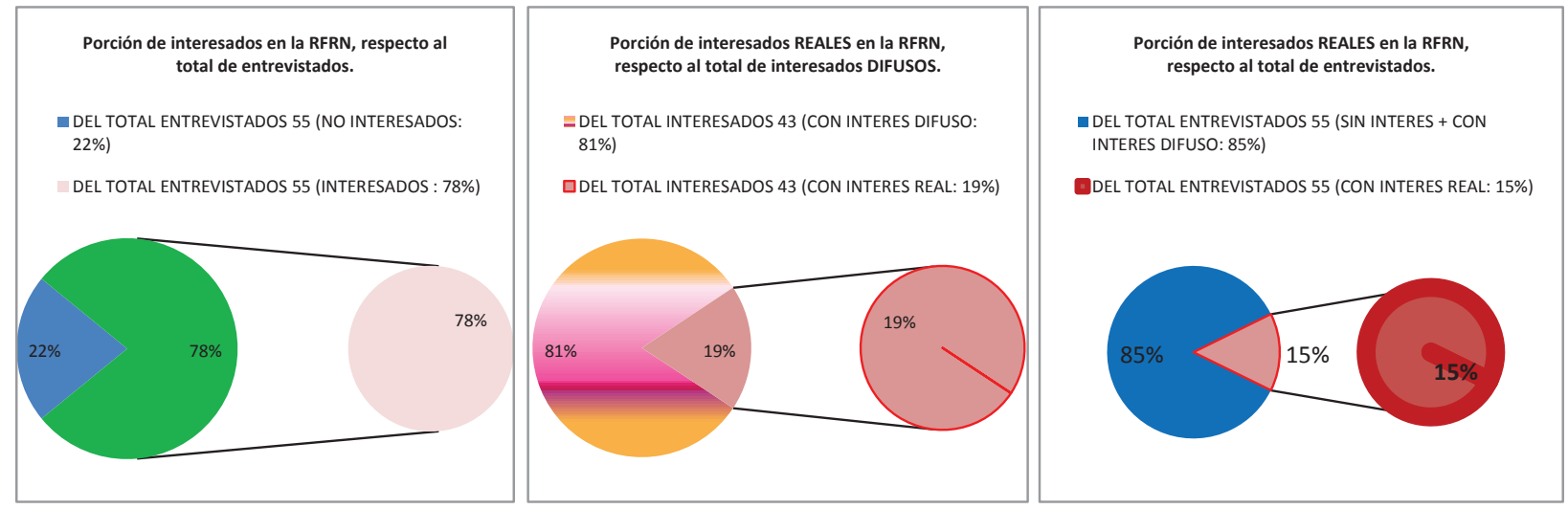

Gráfico 3. Proporciones de los subconjuntos de interesados difusos y reales en la RFRNB.

En el enfoque de Posada Arrubla (2009), el interés existe en la media en que haya conveniencias e inclinaciones; los intereses tienen que ver con la manera de ser, íntima de las personas; tal como piensan y sienten, así son sus intereses; tal como piensa y siente la sociedad, así son sus intereses. Por ello, una sociedad sin formación y sin sentido ambientalista es probable que no tenga algún interés, por lo que está relacionado con el ambiente. La noción de interés, se acerca a lo que se entiende por valor y todo valor surge a partir de un proceso educativo, por ello, lo que se debe entender es la introyección (asimilación) de los valores ambientales de las personas interesadas en el potencial ambiental, de una zona específica.

Al respecto, explica Pérez (2002), que la problemática de los intereses difusos es poder determinar si se trata de una simple suma de intereses individuales o si es una posición de ventaja unitaria o bien, una intermediación entre las dos anteriores. Por ello, la difusión de un interés, se atribuye a un grupo más o menos determinado o indeterminado de sujetos particulares y los intereses son intereses privados de dimensión colectiva.

Resultados mediante lógica difusa: Este método es más tolerante que el tradicional, frente a la clasificación de los intereses de las personas para trabajar en pro de una reserva, considerando que cada persona maneja un alto grado de imprecisión respecto a sus intereses particulares y, más incierto, respecto a sus intereses colectivos, es decir, sus intereses ambientales. Al aplicar la lógica difusa, los grupos o los subconjuntos no se cierran de acuerdo a la mejor posición, sino que se potencia el valor del grupo de interesados, en cuanto a capacidad de aporte. La aplicación de la lógica difusa, en este estudio, se logró en cuatro pasos a saber:

Paso 1: Definición de la inexactitud del interés ambiental: Se definieron las tres variables que ya fueron explicadas (UF, TDLA, DAP), como las que mejor pueden representar los atributos difusos, respecto a los intereses ambientales de la RFRNB.

Paso 2: Asociación de términos lingüísticos: Se establecieron los conjuntos difusos definiendo, por una parte, los contenidos del rango, dentro del cual, cada variable se expresa con inferencias creíbles y, por otra, los límites extremos de cada variable.

Paso 3: Fuzzificación: Para obtener los conjuntos difusos, se asignaron valores numéricos entre 0 y 1 a los atributos de cada variable; luego, se ubicaron las entradas (\# de personas de la muestra que respondieron en este sentido) y, de esta manera, se obtuvieron las tres funciones, en donde $T$ es el conjunto del universo (total de la muestra); UF, TDLA o DAP corresponden en cada caso, al subconjunto que pertenece a $T$ y $X$ representa a los elementos de la valoración, que están entre 0 y 1 . Cada conjunto difuso UF, TDLA o DAP se definió así: 
1) FUNCIÓN DE PERTENENCIA DE UF: $\mathrm{UF}=\{(x, \operatorname{UF}(x)) \mid x \varepsilon \mathrm{T}\}$.

2) FUNCIÓN PERTENENCIA TDLA: $\operatorname{TDLA}=\{(x, \operatorname{TDLA}(x)) \mid x \varepsilon \mathrm{T}\}$.

\section{3) FUNCIÓN DE PERTENENCIA DAP:}

$$
\mathrm{DAP}=\{(x, \operatorname{DAP}(x)) \mid x \varepsilon \mathrm{T}\} .
$$

Los valores asignados a cada función de pertenencia y que fueron incluidos en el sistema computacional utilizado, se presentan en la tabla 2.

Paso 4: Expresión grafica del interés ambiental para cada variable: Las tres variables elegidas y sus resultados, se expresan de manera tridimensional, en el gráfico 4. Aquí, se muestra el comportamiento de las diferentes posiciones, sin descartar ningún grupo de interés en la RFRNB. En estos resultados, se observa que todos los interesados difusos están dentro de la función de pertenencia para potenciar la realidad de la RFRNB, pero se destacan los picos generados por:

- Los valores de quienes no saben exactamente con quien trabajar y de quienes quieren trabajar con pocas personas, lo cual, significa que el trabajo en grupo o las acciones colectivas en la RFRNB son bastante difusas.

- Los valores de quienes no definen el tiempo que pueden dedicarle a la RFRNB y sobresale el grupo de personas que están dispuestas a trabajar indefinidamente, lo que significa que es posible transferir el valor de la Reserva, desde ya, a las futuras generaciones, a través del impulso de quienes manifiestan este tipo de interés.

- El interés por apoyar económicamente a la RFRNB es igual al desinterés por no apoyarla, lo que significa que, en términos de dinero, existen más restricciones y que esta variable es más sensible para demostrar el interés frente a la Reserva.

Con estos resultados, se concluye que para hacer realidad la RFRNB, es necesario que los grupos o el colectivo tengan intereses de hacerlo. Indefectiblemente, los intereses colectivos son intereses difusos, pero no por ello menos importantes, que los intereses individuales reales. Cada grupo de interés tiene un aporte para la RFRNB que debe ser considerado al momento de realizar una gestión socioambiental, como parte del Plan de Manejo Ambiental de la Reserva o de cualquier proyecto ambiental que implique al colectivo social.

En esta vía, Maldonado (2005) reflexiona sobre los intereses difusos y considera que el camino no es fácil y que la construcción social de alternativas aún es posible, considerando que en Colombia se cuenta con un marco jurídico bastante innovador, que considera el derecho-deber de la propiedad, el derecho al ambiente como un derecho colectivo y las plusvalías urbanas.

Adicionalmente, estos resultados obligan a escudriñar en las posibilidades que existen para lograr que la RFRNB sea una

Tabla 2. Valores asignados a cada función de pertenencia, utilizados en el sistema computacional.

\begin{tabular}{|l|c|c|}
\hline \multicolumn{3}{|c|}{ VALORES DE LA FUNCIÓN UNIÓN ESFUERZOS (UF) } \\
\hline ATRIBUTOS UF & VALORES DIFUSOS UF & ENTRADAS UF \\
\hline No sabe & 0 & 38 \\
\hline Solo & 0,2 & 11 \\
\hline Con pocas personas & 0,5 & 35 \\
\hline Con muchas personas & 1 & 16 \\
\hline \multicolumn{2}{|c|}{ VALORES DE LA FUNCIÓN TEMPORALIDAD DE LAS ACCIONES (TDLA) } \\
\hline ATRIBUTOS TDLA & VALORES DIFUSOS TDLA & ENTRADAS TDLA \\
\hline No sabe & 0 & 26 \\
\hline Unos meses & 0,1 & 9 \\
\hline Unos años & 0,4 & 7 \\
\hline Siempre & 1 & 58 \\
\hline \multicolumn{1}{|c|}{ VALORES DE LA FUNCIÓN DISPONIBILIDAD A PAGAR (DAP) } \\
\hline ATRIBUTOS DAP & VALORES DIFUSOS DAP & ENTRADAS DAP \\
\hline No & 0 & 36 \\
\hline No sabe & 0,3 & 46 \\
\hline Sí & 1 & \\
\hline
\end{tabular}


realidad, no solo distrital, sino regional. Estas posibilidades, en primera instancia, están dadas por el potencial de los grupos sociales de querer apoyarla y, otra forma de identificar posibilidades, se encuentra en los instrumentos de planeación del desarrollo territorial, circunscrito al espacio implicado.

Al respecto, Carrizosa (2007) afirma que las variaciones de la complejidad social dependen de las estructuras y de las formas que adopta cada grupo de humanos en un territorio y de las interacciones de esos grupos. Explica que el sistema pasa de ser una muy compleja red de interacciones entre lo rural y lo urbano a un simple objeto del mercado, susceptible de transar por un precio en la ley de oferta y de demanda. En este sentido, se debe buscar, en otros ámbitos, el poder de la RFRNB y, ello se logra, cuando se observa la presión por urbanizarla, no tanto al interior de la zona, sino cuando se evidencia la manera abrupta en que se están urbanizando los municipios de Cota, de Cajicá, de Sopó y de Chía en sus zonas rurales, vecinas a Bogotá y, por ende, vecinas a la RFRNB.

Por otra parte, expresa Maldonado (2009) que, cuando los intereses ambientales ligados al desarrollo urbano se expresan en términos de lógicas rentistas o especulativas, raramente apuntan al interés general, lo cual, hace parte de la esencia de la dimensión ambiental. Por ello, es necesario buscar otras posibilidades de integrar a la declaratoria de la CAR, los instrumentos de la planeación local, con la Distrital y la regional, considerando el eje ambiental como articulador de esta integración. Esta intención institucional, se encuentra en las propuestas que tienen para tal fin la Localidad de Suba (2011), el Distrito Capital de Bogotá (2012), la Gobernación de Cundinamarca (2011) y, en menor medida, pero de gran importancia, los planes de desarrollo y de ordenamiento territorial de los municipios vecinos.
En todo caso, para un análisis de intereses ambientales en una zona de conflicto ambiental, es importante enmarcar las acciones en el pensamiento de Ostrom (2011), quien demuestra cómo en distintos casos, usuarios y propietarios de los bienes comunes han sabido crear instituciones, que permiten el aprovechamiento sustentable. Ella considera que la complejidad de los recursos requiere de sistemas complejos de gobernanza, que involucren la contribución ciudadana de distintas formas. Pero en el caso de la RFRNB, aún el sistema no es visto desde esta perspectiva, la fragmentación de intereses y la red interinstitucional que permite reconstruir el tejido social para, a su vez, proteger el tejido natural, es incipiente.

Para el efecto, Leff (2009) sugiere que la gestión de los recursos naturales implica un proceso de concertación, que permita dirimir conflictos, pero que también abra opciones hacia diferentes estrategias de apropiación y de transformación de la naturaleza, dentro de los principios de racionalidad ambiental. En conclusión, esas estrategias diferentes empiezan por la forma de comprender la situación, que debe ser alternativa a las tradicionales, incluyente y creativa. Objetivo que se cumple, con el hecho de analizar los intereses ambientales, mediante métodos distintos, como la lógica difusa.

Agradecimiento: La autora agradece a la U.D.C.A, por financiar la investigación "Análisis de intereses ambientales en la zona de la RFRNB", que dio origen a este artículo. Conflicto de Intereses: El manuscrito fue preparado y revisado por el autor, quien declara ser el único autor y que no existe ningún conflicto de intereses que ponga en riesgo la validez de los resultados presentados.

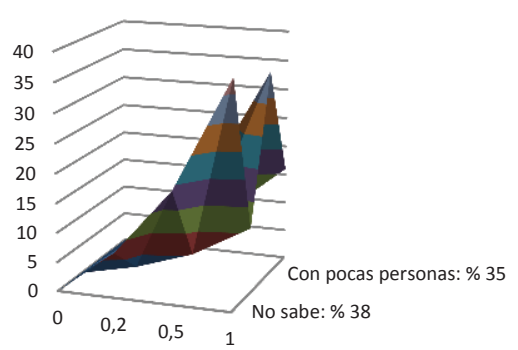

Comportamiento de la variable TEMPORALIDAD DE LAS ACCIONES (TDLA)
Comportamiento de la variable DISPONIBILIDAD A PAGAR (DAP)
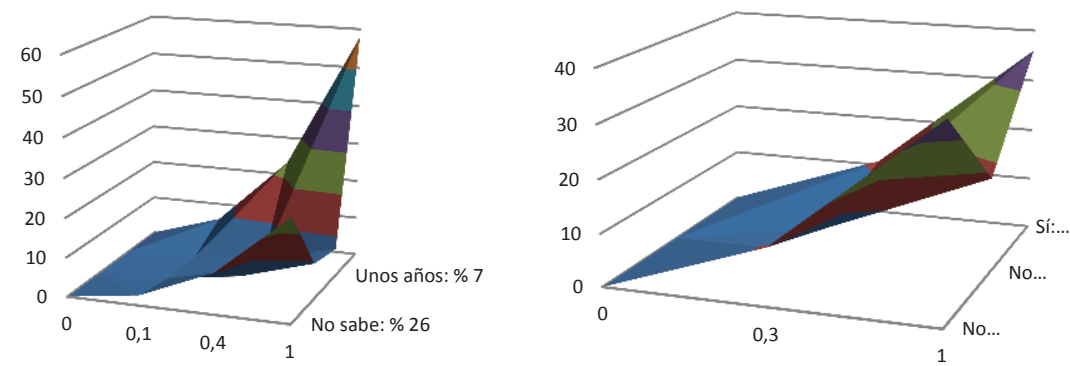

Gráfico 4. Expresión gráfica del interés ambiental en la RFRNB. 


\section{BIBLIOGRAFÍA}

1. ALCALDÍA MAYOR DE BOGOTÁ. 2012. Plan de desarrollo económico y social y de obras públicas para Bogotá distrito capital 2012 - 2016: Bogotá humana. Colombia. 48p.

2. ALCALDIA MAYOR DE BOGOTÁ. 2001. Secretaría Distrital de Ambiente. Agenda ambiental localidad 11, Suba. Colombia. 77p.

3. ALCALDÍA MAYOR DE BOGOTÁ. 2011. Plan de Gestión para el Desarrollo Rural Sostenible, del Distrito de Bogotá. 170p.

4. ALCALDÍA MAYOR DE BOGOTÁ. 2010. Diagnóstico de la región capital Bogotá-Cundinamarca. Colombia 89p.

5. ACEVEDO, J.; CARRIZOSA, J.; CUERVO, L.M.; RODRÍGUEZ, M.; SAMPER, G.; ALDANA, E.; CORREAL, M.; JARAMILLO, R.; SALMONA, R.; VAN DER HAMMEM, T. 2000. Misión de expertos del Ministerio de Ambiente. La zona norte: riqueza ecológica y paisajística que debe ser recuperada y conservada. Mimeógrafo Univerciudad. 9p.

6. ARDILA, G. 2009. Foro Nacional Ambiental. Reflexiones de la mesa de expertos "Bogotá-sabana ¿Gobernabilidad posible? $16 p$.

7. AZNAR R., J.; ALARCÓN R., T. 2006. Etimologías grecolatinas. Pearson Education. México. 224p.

8. AZQUETA, D. 1994. Valoración económica de la calidad ambiental. McGraw-Hill, España. 299p.

9. CARRIZOSA, J. 2006. "Desequilibrios Territoriales y Sostenibilidad Local - Conceptos, metodologías y realidades". Colombia. Ed: Unibiblos. Colombia. 174p.

10. CARRIZOSA, J. 2007. Mente, ambiente y paz. Rev. IDEA. 10:43-52.

11. CORPORACIÓN AUTÓNOMA REGIONAL DE CUNDINAMARCA CAR. 2011. Acuerdo \#011 del Consejo Directivo, mediante el cual declara la "Reserva Forestal Regional Productora del Norte de Bogotá DC Thomas Van der Hammen".

12. DEPARTAMENTO NACIONAL DE PLANEACIÓN DNP. 2010. República de Colombia. Plan nacional de de- sarrollo 2010-2014, "Prosperidad para todos". Tomo I, 541p.

13. DISTRITO BOGOTÁ. 2010. Decreto 043 de enero 29, por el cual se adopta el plan de ordenamiento zonal del norte y se dictan otras disposiciones.

14. D’NEGRI, C.E.; DE VITO, E.L. 2006. Introducción al razonamiento aproximado: lógica difusa. Instituto de Investigaciones Médicas Alfredo Lanari, - CONICET-. Universidad de Buenos Aires. Rev. Argentina Med. Resp. 4:126-136.

15. DURÁN, M.I. (s/f). Lógica Borrosa. Disponible desde Internet en: http://www.it.uc3m.es/jvillena/irc/ (con acceso 08/05/12).

16. DIARIO EL TIEMPO. 2012. Clasificados. Casa Editorial, Bogotá, Colombia. Rev. Metro Cuadrado 105:46.

17. GOBERNACIÓN DE CUNDINAMARCA. 2011. Plan de Desarrollo del Departamento de Cundinamarca 2012-2016.

18. INSTITUTO DE ESTUDIOS URBANOS de la Universidad Nacional de Colombia y Corporación Autónoma Regional-CAR- IEU. 2011. Proyecto borde norte de Bogotá Fase II. 592 p.

19. LOCALIDAD DE SUBA, BOGOTÁ. 2011. Diagnóstico Ambiental. 511p.

20. LEFF, E. 2009. Racionalidad ambiental la reapropiación social de la naturaleza. Siglo XXI editores. Segunda edición. México. 509p.

21. MALDONADO C., M.M. 2005. Son posibles las áreas protegidas alrededor de las grandes ciudades? En: Región, ciudad y áreas protegidas. CEREC. p.181221.

22. MALDONADO C., M.M. 2009. Foro Nacional Ambiental. Reflexiones de la mesa de expertos "Bogotá-sabana ¿Gobernabilidad posible? $16 \mathrm{p}$.

23. MENDIETA L., J.C. 2005. Manual de valoración económica de bienes no mercadeables: Aplicaciones de las técnicas de valoración no mercadeables y el análisis costo beneficio y medio ambiente. Uniandes, CEDE, Colombia. 338p.

24. MORALES L., G. 2002. Introducción a la lógica difusa. Centro de Investigación y Estudios Avanzados. México. 
25. OSTROM, E. 2011. El Gobierno de Los Bienes Comunes: La Evolución de Las Instituciones de Acción Colectiva. Fondo de Cultura Económica México. $2^{\mathrm{a}}$ ed. 403p.

26. PECHE G., R. 2006. Valoración cualitativa de impactos ambientales mediante lógica borrosa. Rev. Gestión y ambiente. 9(3):99-113.

27. PÉREZ C., L. 2002. La defensa judicial de los intereses ambientales. Ediciones Lex Nova. España. 428p.

28. POSADA L., L.G. 1997. La problemática ambiental y los diversos enfoques de la teoría económica. Rev. Ens. Econ. 7:32-52.

29. POSADA A., A. 2009. Intereses ambientales: reflexiones sobre la aplicación de lo difuso. Rev. Ing. 8(14):1120.
30. PROCURADURÍA GENERAL DE LA NACIÓN PGN. 2010. Circular \#023 de abril 13, sobre el pronunciamiento respecto a la reserva forestal protectora del norte y el plan de ordenamiento zonal del norte.

31. ROMERO, A. 2012. Mapa en SIG del proyecto Análisis de intereses ambientales en la RFRNB, sobre la base cartográfica del proyecto institucional U.D.C.A

Recibido: Agosto 21 de 2012

Aceptado: Octubre 26 de 2012 